\title{
Hippocampal Damage and Exploratory Preferences in Rats: Memory for Objects, Places, and Contexts
}

\author{
Dave G. Mumby, ${ }^{1}$ Stephane Gaskin, Melissa J. Glenn, Tania E. Schramek, \\ and Hugo Lehmann \\ Center for Studies in Behavioral Neurobiology, Department of Psychology, Concordia University, Montreal, H4B 1R6, Quebec, Canada
}

\begin{abstract}
Rats have a natural tendency to spend more time exploring novel objects than familiar objects, and this preference can be used as an index of object recognition. Rats also show an exploratory preference for objects in locations where they have not previously encountered objects (an index of place memory) and for familiar objects in contexts different from those in which the objects were originally encountered (an index of context memory). In this experiment, rats with cytotoxic lesions of the hippocampal formation were tested on all three versions of the novelty-preference paradigm, with a 5-min retention interval between the familiarization and test phases. Rats with sham lesions displayed a novelty preference on all three trial types, whereas the rats with hippocampal lesions displayed a novelty preference on Object trials but did not discriminate between the objects on Place trials or Context trials. The findings indicate that hippocampal damage impairs memory for contextual or spatial aspects of an experience, whereas memory for objects that were part of the same experience are left relatively intact.
\end{abstract}

Findings from lesion studies indicate that the integrity of the hippocampal formation (HPC) is essential for normal memory of certain types of information about an event, whereas other information about an event may be remembered in a relatively normal way even when hippocampal damage is extensive or complete. For example, the HPC is widely thought to play a critical role in remembering the environmental context in which an event occurs (Hirsch 1974; Kim and Fanselow 1992; Eichenbaum et al. 1994; Nadel and Moscovitch 1997) and where an event occurs within allocentric space (Eichenbaum 2000; O'Keefe and Nadel 1978). In contrast, the findings from several lesion studies indicate that the HPC is not essential for recognizing the objects that were part of a previous event (Steckler et al. 1998; Aggleton and Brown 1999; Mumby 2001).

One shortcoming of the evidence for these contentspecific dissociations in memory after HPC damage is that it is mostly derived from comparing findings across studies that varied widely in the extent of the HPC damage (Jarrard 2001) and the types of behavioral paradigms used to assess memory. For example, evidence that the HPC plays a critical role in remembering places comes from studies showing that rats with HPC damage are impaired on allocentric-spatial memory tasks in water mazes (Morris et al. 1982; Sutherland et al. 1982) and radial-arm mazes (e.g., Kesner et al.

${ }^{1}$ Corresponding author:

E-MAIL mumby@vax2.concordia.ca; FAX 514-848-4545.

Article and publication are at http://www.learnmem.org/cgi/doi/ $10.1101 / \mathrm{lm} .41302$.
1993). Much of the evidence that HPC damage impairs memory for contextual information comes from reports of impaired contextual fear conditioning after HPC damage (Kim et al. 1993; Young et al. 1994; Maren and Fanselow 1997). Evidence of preserved object-recognition abilities comes from several studies that found little or no impairment on object-based delayed nonmatching-to-sample (DNMS) tasks in rats with HPC or fornix damage (reviewed by Aggleton and Brown 1999; and Mumby 2001), although there have been a few exceptions (Wiig and Bilkey 1995; Clark et al. 2001).

These tasks differ in several ways other than just the type of information that must be remembered. Other differences include the nature of the motivation or reward, the reinforcement contingencies, and the amount of training required. The behaviors that are measured to assess memory also vary considerably and include conditioned reflexes (e.g., Pavlovian fear conditioning), speed or accuracy of spatial navigation, which can involve either swimming (water maze) or running (radial maze), and manipulation or displacement of objects (e.g., delayed nonmatching-tosample). Any of these variables could potentially interact with the functional consequences of HPC damage in ways that produce a misleading picture of how well various aspects of an event are remembered after HPC damage.

Another factor that weakens the conclusions that can be drawn about content-specific memory deficits from tasks that use such diverse procedures and behavioral variables is that the episodes about which the rat has to remember

LEARNING \& MEMORY 9:49-57 @ 2002 by Cold Spring Harbor Laboratory Press ISSN1072-0502/02 \$5.00

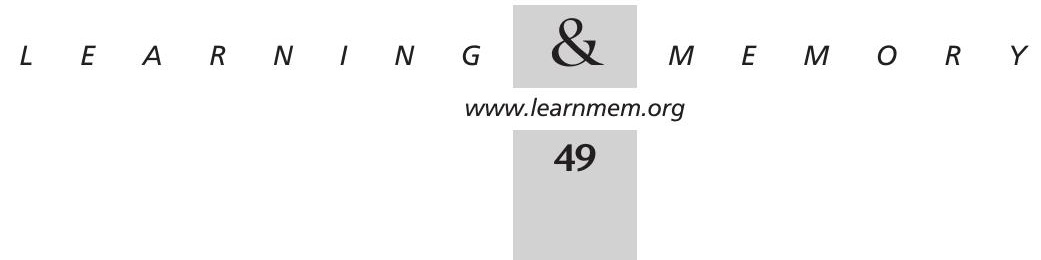


something entail vastly different experiences. For example, the experience of encountering an object during the sample phase of a delayed nonmatching-to-sample trial is very different from the experience of bumping into a hidden platform while swimming in a pool of cool water. Both experiences occur in a particular context and place, and both involve objects, and it is presumed that normal rats remember these content-specific aspects of both experiences for some time thereafter. But, differences in the learning conditions in these two examples might influence the way that elements of those events are represented in the brain and the extent to which remembering them later depends on the HPC. One likely mechanism for such an influence could involve differences in the level of arousal or stress induced by different training conditions.

The aim of the present experiment was to determine whether HPC damage would affect memory for contextual or spatial aspects of an event while sparing the ability to recognize objects that were part of the same event. To assess object recognition, we used a novelty-preference paradigm that was originally developed for this purpose by Ennaceur and Delacour (1988). In the conventional version of this task, a rat is placed in an open-field arena and allowed to explore two identical sample objects for a few minutes. The rat is then removed from the arena for a retention delay, after which it is returned to the arena with two new objects-one is identical to the sample and the other is novel. Normal rats spend more time exploring the novel object during the first few minutes of the retention test, and when this bias is observed it is inferred that the rat recognizes the sample object. Rats with fornix lesions display a preference for the novel object after retention delays of up to several minutes (Ennaceur and Aggleton 1994; Ennaceur et al. 1996, 1997; Warburton and Aggleton 1999), which indicates that the HPC is not essential for object recognition in this paradigm. However, it was recently reported that cytotoxic lesions of the HPC caused deficits after a 24-hr retention delay (Clark et al. 2000), although rats in the same study that had fornix lesions or radiofrequency lesions of the HPC performed normally after the 24-hr delay.

A different version of the novelty-preference paradigm requires rats to recognize that an object is in a place where there had not previously been an object (Ennaceur et al. 1997). On this place-memory version, rats are familiarized with two objects, and for the retention test one of them is moved to a novel location within the arena-a location where an object has never before been encountered. Normal rats spend more time exploring the object in the novel location than the one in the familiar location, but rats with fornix lesions do not show a preference (Ennaceur et al. 1997).

Another version of the novelty-preference paradigm has been used to test rats' ability to remember that an object was previously encountered in a particular context (Dix and Aggleton 1999). Rats were familiarized with two different objects, each in a unique context (i.e., different open fields), and exploratory preference was later measured on a test in which both objects were presented in one of the two contexts. Rats spent more time exploring the object that was in a context different from that during familiarization. One goal of the present experiment was to determine the effects of HPC damage on performance of this contextmemory version.

In recent years the novelty-preference paradigm has become a favored method for assessing object recognition in rats, mainly for practical reasons. Because it is used to measure a spontaneous preference, subjects do not need to be deprived of food or water, nor do they require extended training. The same advantages apply to the place- and context-memory versions. The same simple materials and general procedures are used for all three versions, and because they do not require the learning of a contingency rule, trials can be administered to each subject repeatedly. Thus, interpretation of results should not be complicated by procedural learning, provided that the intertrial intervals are sufficiently long.

Another advantage is that the familiarization phase is identical for all three versions (with the exception that there are two familiarization phases on the context-memory task); this means that the different versions assess memory for different aspects of what are essentially equivalent events $-5 \mathrm{~min}$ in the arena with two identical objects. The manipulation that sets the different versions apart occurs on the retention test, thus there is no confounding of memory for different types of information with the nature of the learning events.

\section{RESULTS}

\section{Histological Results}

Figure 1 illustrates the extent of the hippocampal lesions. The NMDA injections produced extensive cell loss in all principle subfields of the hippocampus and dentate gyrus. However, there was sparing of cells in the most ventral CA1 subfield unilaterally in one rat and bilaterally in two rats. Cells in the dorsal lateral CA2 and CA3 fields were also spared unilaterally in one rat. There was variable bilateral loss of cells in the subiculum of each rat. There was also significant damage to the fimbria/fornix in two rats, but this structure was generally spared in the others. Two rats had unilateral cell loss in the lateral posterior nucleus of the thalamus, but no other damage was found in the thalamus. All rats had some thinning of the parietal cortex where the injection cannulae were inserted. In one rat there was unilateral cell loss in the medial part of the temporal cortex and perirhinal cortex, but these areas, along with the entorhinal cortex, showed no evidence of damage in the remaining rats.

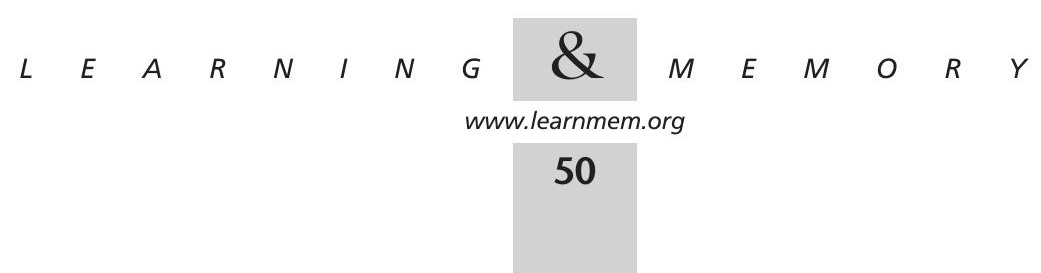



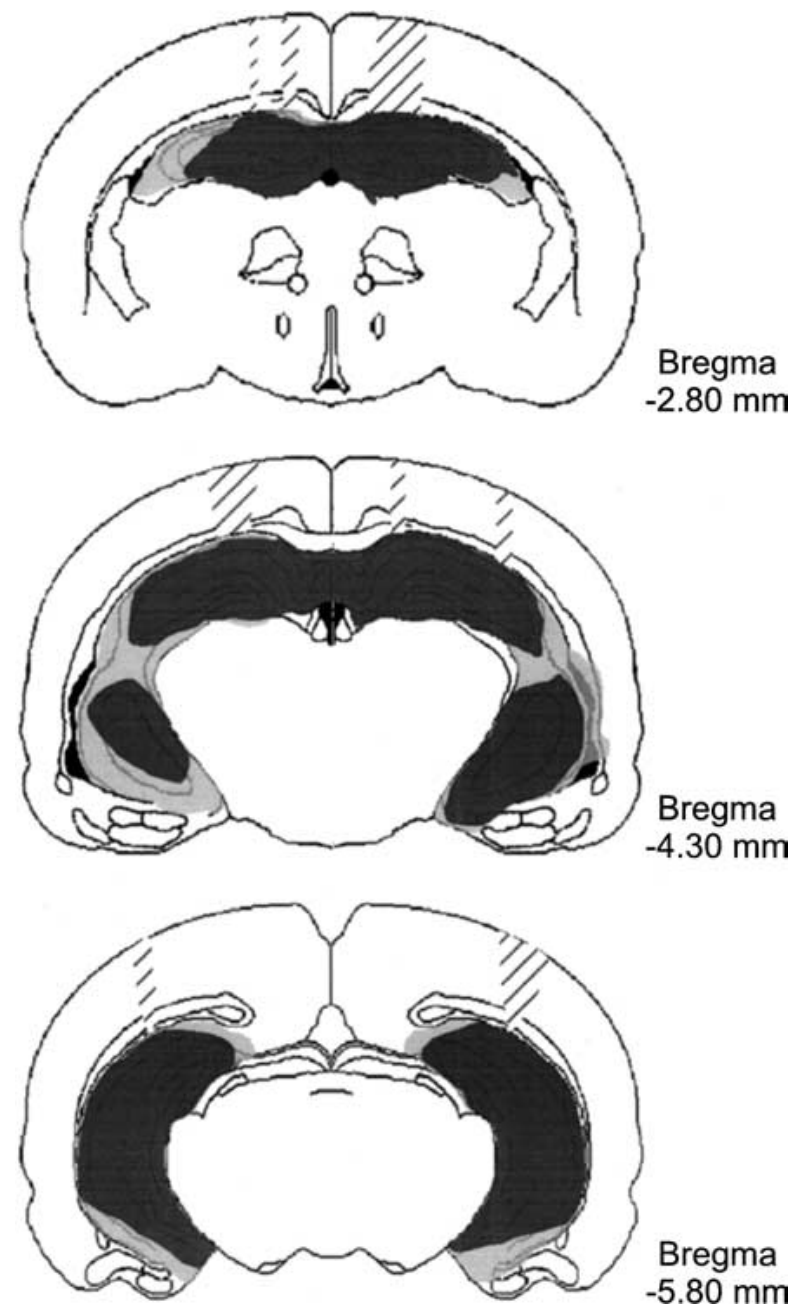

Figure 1 Illustration of the smallest (black) and largest (grey) hippocampal lesions observed at three coronal planes (distance in millimeters from bregma). Cross-hatching represents cortical damage.

\section{Behavioral Results}

Figure 2 shows the time spent exploring objects during the familiarization and test phases for each trial type. There were no significant differences between the groups or among the versions during the familiarization phase. For the test phase, there was a significant effect of Version $(F[2,50]=15.78, p<.001)$ but nonsignificant effect of Lesion and a nonsignificant interaction. Both groups displayed the most object exploration during the test phase on Object trials and the least on Context trials. Because there were no significant group differences, these data indicate that any significant differences between the groups in terms of discrimination on the test phase does not result from differences in time spent exploring the sample objects during the familiarization phase or from differences in total object exploration during the test.

Figure 3 shows the mean exploration ratios during the test phase for each version. The ratios indicate the degree of discriminated exploration of the sample and target objects, taking into account variations in exploration times across rats and trials. Data are shown separately for the first minute, the second minute, and the third minute of the test phase, and also for all $3 \mathrm{~min}$ of the test phase.

One-sample $t$ tests were used to determine whether the exploration ratios under each condition were significantly different from chance levels. The asterisks in Figure 3 indicate the results of this analysis. For sham rats, ratios based on the total 3-min test were significantly above chance on all three trial types; whereas for HPC rats, they were significantly above chance on only the Object trials. For sham rats, the minute-by-minute ratios were above chance in the first and second minute on Object and Place trials, and in the first minute on Context trials. The HPC rats' ratios were significantly above chance in the first and second minute on Object trials but not in any of the time bins on Place or Context trials.

An ANOVA performed on the exploration ratios for the total 3-min test phase yielded significant main effects of Lesion $(\mathrm{F}[1,25]=12.34, p<.002)$ and Version $(\mathrm{F}[2,50]=7.86$, $p<.001)$ and a nonsignificant interaction $(\mathrm{F}<1)$. Planned comparisons revealed that HPC rats had exploration ratios that were significantly lower than those of sham rats on Place trials $(\mathrm{t}[25]=2.24, p=.034)$ and Context trials $(\mathrm{t}[25]=2.93, p=.007)$, but the groups did not differ significantly on Object trials $(\mathrm{t}[25]=1.00, p=.325)$.

The minute-by-minute data show how discriminated object exploration changed over the test phase. An ANOVA performed on the whole dataset revealed significant main effects of Lesion $(\mathrm{F}[1,25]=12.53, p<.001)$ and Version $(\mathrm{F}[2,50]=8.14, p<.001)$ and a nonsignificant main effect of Minute $(\mathrm{F}[2,50]=2.15, p>.10)$. None of the interactions

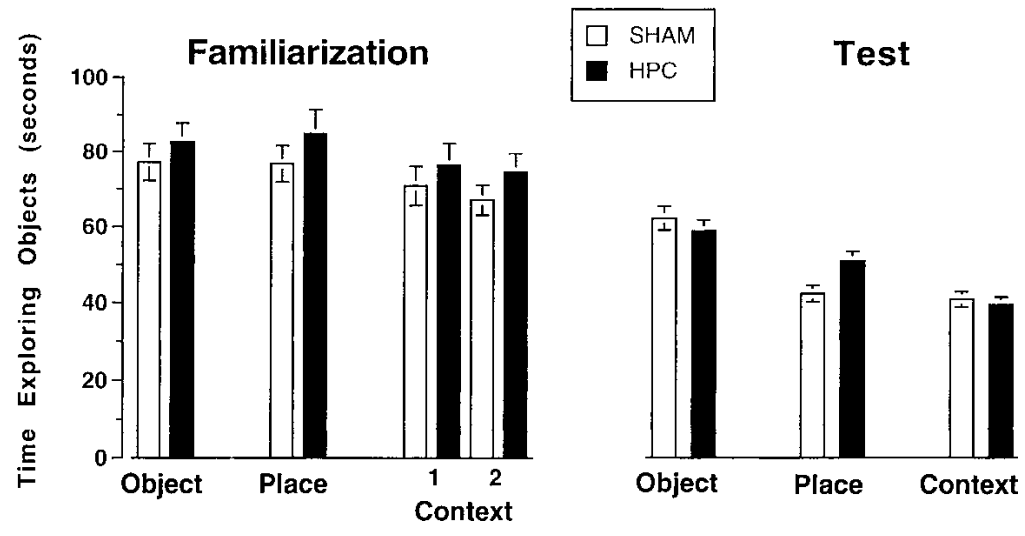

Figure 2 Time engaged in object exploration during the familiarization and test phases for each trial type. For Context trials, data are shown separately for the first (1) and second (2) familiarization phases. Error bars represent SEM.

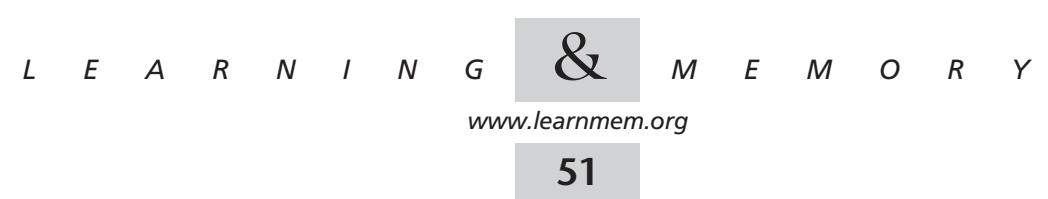



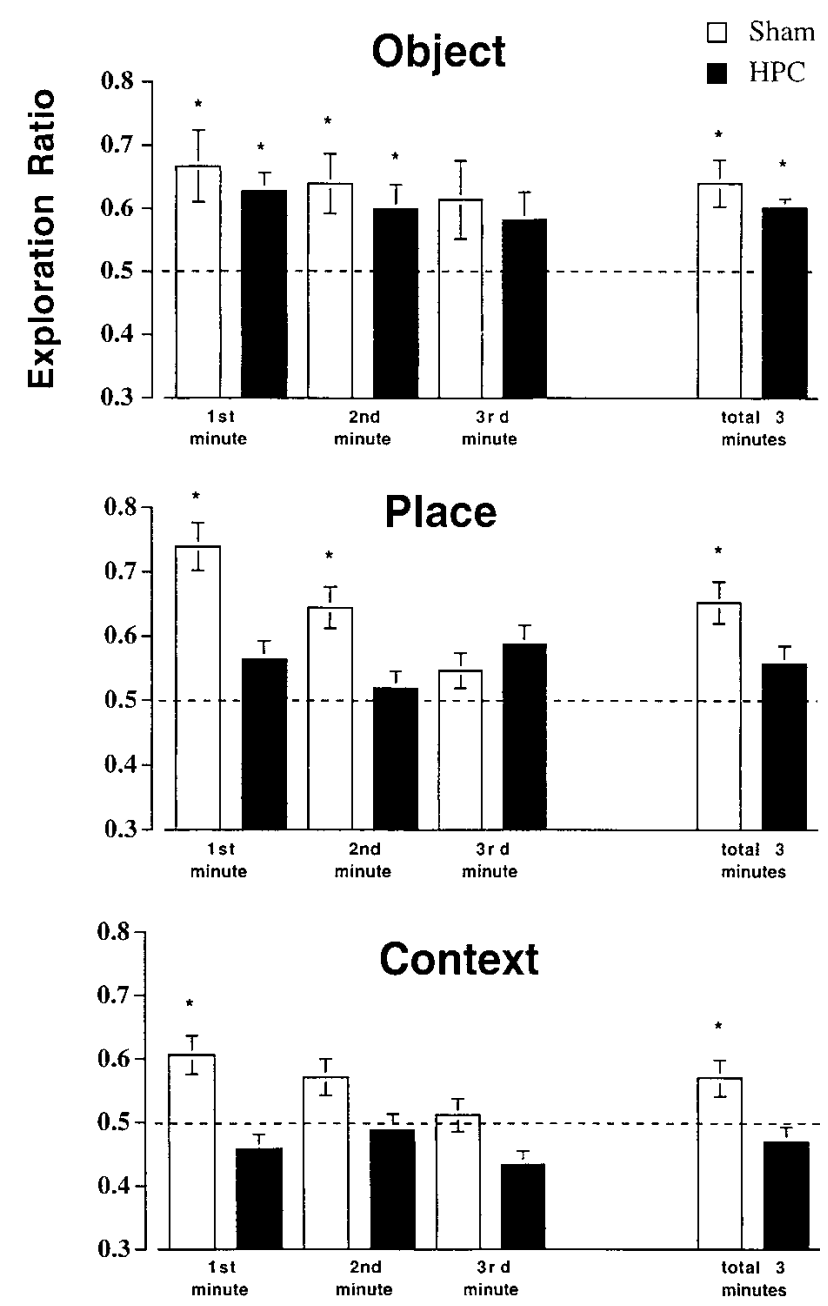

Figure 3 Mean exploration ratios during the retention test for each trial type. The ratio represents the proportion of object-exploration time that was spent exploring the novel object; $t_{\text {novel }} /$ $\left(t_{\text {novel }}+t_{\text {sample }}\right)$. The dashed line represents the chance level of performance (i.e., a ratio of 0.50 ; no discrimination between sample and target objects). Data are shown separately for the first, second, and third minute of the test and for all 3 min of the test combined. Asterisks denote mean ratios that are significantly above chance (one-sample $t$ tests, $p<.05$ ). Error bars represent SEM.

were statistically significant. The data from the two groups were then analyzed separately. For sham rats, there were marginally significant effects of Version $(\mathrm{F}[2,26]=3.275$, $p=.054)$ and Minute $(\mathrm{F}[2,26]=3.209, p=.057)$ and a nonsignificant interaction. The sham rats' exploration ratios tended to decrease across the $3 \mathrm{~min}$ of the retention test; overall, this linear trend was statistically significant $(\mathrm{F}[1,13]=5.26, p<.05)$. When the data from each version were analyzed separately, the linear trend was significant on only the Place trials $(\mathrm{F}[1,13]=19.44, p<.001)$. For HPC rats, there was a significant effect of Version $(F[2,24]=4.98$, $p<.02$ ) but a nonsignificant effect of Minute and a nonsignificant interaction (both Fs $<1$ ). The HPC rats displayed no consistent change in ratios across the test phase, either overall or for any version analyzed separately (all ps > .10).

On Context trials, the test context could be either the first or second context from the familiarization phase. The Context data were reevaluated to determine whether this factor had an impact on the exploration ratios based on the total 3-min test. In sham rats, the mean ratio on trials using the first-presented and second-presented context as the test context was $.557(\mathrm{SE}=.024)$ and $.574(\mathrm{SE}=.044)$, respectively; for HPC rats, the corresponding means were .478 $(\mathrm{SE}=.044)$ and $.427(\mathrm{SE}=.047)$. Formal statistical analyses were not performed on these data because the test context (first or second) was not counterbalanced within subjects (each rat received three Context trials), and the groups were not matched for the proportion of rats having more tests with the first-presented or second-presented context (because we chose instead to equate them for the proportion of tests that were in Room A or Room B). However, the proximity of the mean ratios within each group, and the degree of the variance around them, indicates that the test context (first or second presented) made little difference to the performance of either sham or HPC rats.

\section{DISCUSSION}

The main findings were that control rats spent more time exploring the target than the sample object on all three trial types, whereas rats with HPC lesions displayed a similar preference for the target on Object trials but did not discriminate between the objects on Place trials or on Context trials. Between-group comparisons confirmed that HPC rats were impaired on the Place and Context trials but not on Object trials. These results indicate that the HPC plays an essential role in remembering where and in what context an object is encountered but makes a more expendable contribution to object recognition (i.e., discriminating the familiarity of a previously encountered object). Alternative explanations for the results are limited because the learning episode was equivalent for all three trial types, which eliminates the potential for confounding of content-specific memory with either known or unknown variables that occur when different procedures are used to assess different types of memory.

For the familiarization phase of each trial, the rat was allowed to explore two identical objects in the arena for 5 min. With only subtle changes to those conditions, the retention test specified what aspect of the familiarization phase had to be remembered to show preferential exploration of the target object. Again, the rat was allowed to explore two objects, one of which was just as it had been during the familiarization phase, but the other object was either replaced with a novel object, moved to a novel location, or replaced with a familiar object that had been encountered in a different context. To show a reliable prefer-

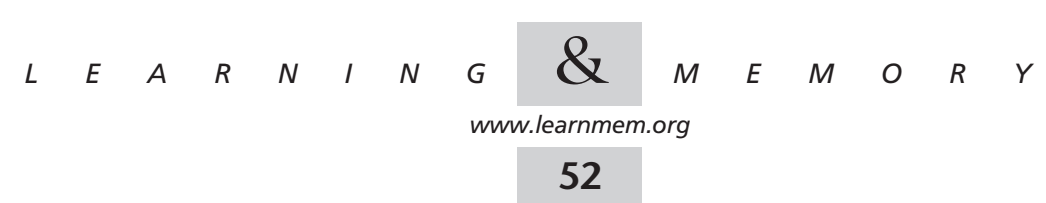


ence for the target, the rats had to recognize the sample object on Object trials and they had to remember where objects are normally positioned on Place trials and the context in which a particular object had been previously encountered on Context trials.

It seems likely the dissociation observed in HPC ratsnormal performance on Object trials but deficits on Place and Context trials-reflects a disproportionate effect of the lesions on memory for the contexts or places in which objects were encountered, with little or no effect on memory for the objects themselves. The dissociation cannot be attributed to variability in the lesions because the dissociation occurred within subjects. It cannot be attributed to differences in the extent to which sham and HPC rats explored the objects during the familiarization phase or to differences in the overall amount of object exploration during the test phase, because the groups did not differ significantly on those measures.

The ability of rats with HPC lesions to discriminate between the sample and target on Object trials rules out several explanations for their failure to discriminate on Context trials. The deficits on Context trials were not attributable to perceptual impairment in discriminating the features of the objects or an inability to recognize them. The results on Object trials also indicate that the HPC lesions did not disrupt the rats' instinctive bias for exploring novelty. It is more likely that the lesions disrupted either the formation or the retrieval of associations involving the objects and the particular contexts in which they were encountered. However, it is also possible that it was the contexts themselves that were forgotten. If so, the context in which the retention test occurred would be irrelevant. The rat would encounter two familiar objects and there would be no reason to explore them differently.

The results on the Context trials are consistent with evidence from other paradigms indicating that HPC damage impairs memory for contextual stimuli or for associations between events and the environmental contexts in which they occur. Rats with hippocampal lesions remember discrete cues that are paired with shock during Pavlovian fear conditioning but are impaired at remembering the context in which the shocks occurred (Kim and Fanselow 1992; Kim et al. 1993; Young et al. 1994; Maren and Fanselow 1997). It is also important to note that on Context trials the target object appears in a new place, as well as a new context, and this might be the element of novelty that normally supports discriminated object exploration on this version, not the context-mismatch.

The results of the Place trials are consistent with previous evidence that the HPC is needed for remembering where objects are located in allocentric space. In one study, normal rats explored the location where an object had been removed from a familiar environment, but rats with dorsal HPC lesions did not (Save et al. 1992). In another study, rats with fornix lesions were impaired on the same placememory version of the novelty-preference paradigm that we used in the present experiment (Ennaceur et al. 1997).

The results on Place trials are also consistent with other evidence of impaired allocentric-spatial information processing in rats with HPC lesions on tasks using water mazes, radial-arm mazes, or other mazes where the subject must navigate to the location of a goal item or reward. One explanation that has been proposed for the deficits displayed by rats with HPC damage on water-maze and radial-armmaze tasks is that those deficits could be attributable to impaired navigational abilities, rather than simply impaired place representation (Redish and Touretzky 1997; Whishaw et al. 1997). Such an explanation cannot easily account for the deficits our HPC rats showed on Place trials because there is no navigational component to this task.

It should be noted that Place trials differed from Object and Context trials in that the rat could discriminate between the target and sample on Place trials even without remembering anything from the familiarization phase of the same trial. For each rat, objects were encountered in the standard locations on 52 occasions throughout the experiment (including the objects that were present during habituation to the arenas), but in novel locations only three times. Presumably, the rats learned over several trials to expect objects to appear in the standard positions. Therefore, it is not clear whether normal rats discriminate on Place trials because they recognize that the target object is in a different place on the retention test than it was during the familiarization phase or that it is in a different place from all or most other objects the rat has encountered on previous trials.

On Object trials, the HPC rats displayed a significant preference for the novel object, and the magnitude of that preference was similar to that of sham rats. These results provide no evidence of impaired object-recognition memory after extensive HPC damage in rats. One could argue that deficits might have occurred if the retention delays were longer than the 5-min delays used in this experiment. But recent findings indicate that performance is often normal after delays of up to $24 \mathrm{hr}$ in rats with HPC damage. Although there is a report of deficits after a 24-hr delay in rats with ibotenic acid lesions of the HPC (Clark et al. 2000), rats in the same study that received either radiofrequency HPC lesions or fornix lesions performed at levels similar to control rats at the 24-hr delay. A more recent study found no deficits after delays of $15 \mathrm{~min}$ or $24 \mathrm{hr}$ in rats with NMDA lesions of the HPC like those in the present experiment ( $\mathrm{S}$. Gaskin and D.G. Mumby, in prep). The unreliability of deficits with such long retention intervals is problematic for the view that the HPC plays an essential role in object recognition in rats.

Other evidence for spared object-recognition memory after HPC or fornix damage in rats comes from several stud-

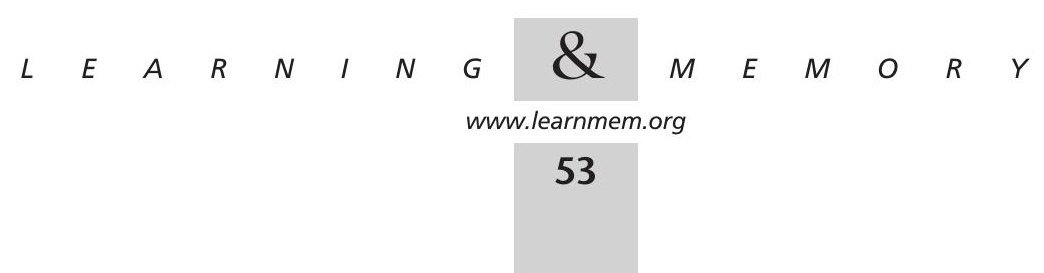


ies that used object-based DNMS tasks (Aggleton et al. 1986; Rothblat and Kromer 1991; Jackson-Smith et al. 1993; Kesner et al. 1993; Rawlins et al. 1993; Shaw and Aggleton 1993; Yee and Rawlins 1994; Glenn and Mumby 1996; Mumby et al. 1996; Cassaday and Rawlins 1997; Duva et al. 1997). The data are not entirely consistent, however, because DNMS deficits have been reported in a small number of studies (Clark et al. 2001; Mumby et al. 1992; Mumby et al. 1995; Wiig and Bilkey 1995), leading some to conclude that object-recognition is impaired by HPC damage (Clark et al. 2001). A recent review of studies examining this question concluded that the evidence for impaired object-recognition memory was questionable in at least two of these latter studies (Mumby et al. 1992, 1995) and depended on which analysis was performed on the data (Mumby 2001). In sum, the preponderance of evidence from rats indicates that the hippocampus plays, at most, a very limited role in object recognition, and perhaps none at all. A similar conclusion was reached in recent reviews of neuroanatomical studies of spatial and nonspatial recognition in rats (Steckler et al. 1998; Aggleton and Brown 1999; Duva and Pinel 1999).

Although the rats with HPC lesions in the present study provided evidence of a content-specific dissociation in memory, the sham rats provided some insight into the exploratory tendencies of rats encountering various kinds of changes within their environments. It has been previously reported that rats' tendency to discriminate between the sample and target objects on the object-recognition version diminishes after the first 2 min of the test trial, presumably because the difference in familiarity between the two objects diminishes as the trial progresses (Dellu et al. 1992; Dix and Aggleton 1999; Mumby 2001). Rats in one study also displayed rapid habituation of novelty exploration on place-memory and context-memory trials like those used in the present experiment (Dix and Aggleton 1999). Our present results confirm those findings. Overall, the exploration ratios of the sham rats decreased significantly during the 3 min of the test phase, and on each version they failed to discriminate between the sample and target objects during the third minute of the test.

The findings of this experiment extend the range of tasks that can be used to show preserved object-recognition memory in rats with extensive hippocampal damage whereas showing that the same damage impairs memory for contextual information and also for spatial location. An important feature of the novelty-preference paradigm is that the learning conditions are equivalent for the different versions, and the retention tests are also very similar. These similarities strengthen the validity of comparisons of memory for different types of information after brain lesions or other treatments, because many potential confounds are eliminated that could otherwise obscure or distort the status of content-specific memories.

\section{MATERIALS AND METHODS}

\section{Subjects}

The subjects were 27 experimentally naive, male, Long-Evans rats (Charles River) between approximately 10 and $12 \mathrm{wk}$ old at the beginning of the experiment. They were housed individually with continuous access to food and water under a $12: 12$ light-dark cycle, with light onset at 8:00 a.m.

\section{Surgery}

Surgery was performed under pentobarbitol anesthesia $(65 \mathrm{mg} / \mathrm{kg})$. HPC lesions $(n=13)$ were made bilaterally by intrahippocampal injections of a $5.1 \mathrm{M}$ solution of N-methyl-D-aspartate (NMDA) dissolved in $0.1 \mathrm{M}$ phosphate buffered saline. Injections were made at 10 sites bilaterally with $10-\mu \mathrm{L}$ Hamilton syringes mounted in an infusion pump (KD Scientific) and connected to 30-gauge cannulae by polyethylene tubing. Table 1 shows the injection coordinates. The NMDA solution was infused at a flow rate of $.15 \mathrm{~mL}$ per min. until a total of $.4 \mathrm{~mL}$ had been injected at each site. The cannulae were left in place for an additional $2.5 \mathrm{~min}$ before being retracted. The scalp incision was closed with wound clips and an antibiotic powder was applied to the wound. As the rats began awakening from the anesthetic, they were given a dose of diazepam (approximately $10 \mathrm{mg} / \mathrm{kg}$ ) as a prophylaxis against seizures. Rats in the sham group $(n=14)$ received the same anesthetic dose, scalp incision, and postsurgery diazepam injections, but no damage was done to the skull or brain. Rats were allowed to recover for $14 \mathrm{~d}$ before training commenced.

\section{Apparatus}

Two open-field arenas $(60 \mathrm{~cm} \times 70 \mathrm{~cm} \times 70 \mathrm{~cm})$ constructed of grey PVC plastic were used. Each arena was situated in a different room. The two rooms differed in several ways that could be easily detected by the rats from inside the arena, including the appearance of the ceiling and walls and ambient illumination. Strips of colored cardboard lined the top of the walls in one of the arenas to provide another salient feature that distinguished the two arenas from each other. Thus, the two arenas, and the testing rooms they were located in, constituted two different contexts.

All other features of the arenas were the same. A stainless-steel tray served as the floor and was covered with wood shavings. The

Table 1. Cannulae Coordinates Relative to Bregma (in millimeter), for NMDA Lesions of the Hippocampal Formation

\begin{tabular}{lcc}
\hline $\begin{array}{l}\text { Anteroposterior } \\
(\mathrm{AP})\end{array}$ & $\begin{array}{c}\text { Mediolateral } \\
(\mathrm{ML})\end{array}$ & $\begin{array}{c}\text { Dorsoventral } \\
(\mathrm{DV})\end{array}$ \\
\hline-3.1 & \pm 1.0 & 3.6 \\
-3.1 & \pm 2.0 & 3.6 \\
-4.1 & \pm 2.0 & 4.0 \\
-4.1 & \pm 3.5 & 4.0 \\
-5.0 & \pm 3.0 & 4.1 \\
-5.0 & \pm 5.2 & 5.0 \\
-5.0 & \pm 5.2 & 7.3 \\
-5.8 & \pm 4.4 & 4.4 \\
-5.8 & \pm 5.1 & 6.2 \\
-5.8 & \pm 5.1 & 7.5 \\
\hline
\end{tabular}

NMDA, N-methyl-D-aspartate.

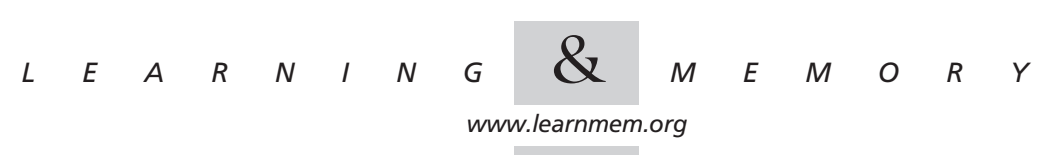


floor could be removed through a slot at the bottom of one wall to facilitate changing the shavings between each trial. A videocamera was positioned over the arena and sample and test phases were videotaped for later analysis.

The 18 objects that served as stimuli were made of metal, glass, porcelain, or glazed ceramic. There were three or four copies of each object, and the copies were used interchangeably. The objects varied in height between approximately $4.5 \mathrm{~cm}$ and $15 \mathrm{~cm}$, and in width between approximately $4 \mathrm{~cm}$ and $10 \mathrm{~cm}$. The objects were washed after every trial.

Figure 4 shows the positions of the objects within the arenas. Attached with epoxy to the bottom of each object was a small glass jar ( $6 \mathrm{~cm}$ high); attached to the floor of the arena, at the locations shown in Figure 4, were two inverted jar lids. Objects were fixed in place by screwing the jars into the lids. Standard positions were used for the Object and Context trials and for the familiarization phase of Place trials. The standard positions were $27 \mathrm{~cm}$ from opposing corners of the arena. There were two "novel" positions in each arena to which one of the objects could be moved for the retention test on Place trials; the novel positions were $18 \mathrm{~cm}$ from the other two opposing corners of the arena.

\section{Procedure}

Figure 4 illustrates the three types of novelty-preference trials. On Object trials, the rat was placed into the arena with two identical sample objects and allowed to explore for $5 \mathrm{~min}$. The rat was then removed and the objects were replaced with two new objects; one of the new objects was identical to the sample and the other was a novel object that the rat had never before encountered. The rat was returned to the arena for the retention test and allowed to explore for $3 \mathrm{~min}$.

On Place trials, the rat was placed into the arena with two identical objects and allowed to explore for $5 \mathrm{~min}$. The rat was then removed for the retention interval, and one of the objects was moved to a new location (Fig. 1). The rat was returned to the arena and allowed to explore for $3 \mathrm{~min}$.

On Context trials, the rat was placed into an arena with two identical objects and allowed to explore for $5 \mathrm{~min}$. The rat was removed and carried to a second arena located in another room where it was allowed to explore a new pair of objects for $5 \mathrm{~min}$. The rat was then removed for the retention interval. On the retention test, a copy of each object was presented in one of the two contexts.

On all trials, the familiarization phase was $5 \mathrm{~min}$, the retention interval was $5 \mathrm{~min}$, and the retention test was $3 \mathrm{~min}$. Rats spent the retention interval in a plastic carrying box in the colony room. For ease of description, the term "target" object is used to refer to the novel object on Object trials, the moved object on Place trials, and the context-mismatched object on Context trials; the other object is referred to as the "sample" for all three trial types.

The rats were first habituated to the arenas by allowing them to explore each arena for three daily 20-min sessions. Two identical objects were present in the arenas during habituation sessions; those objects were not used in the subsequent experimental trials.

Experimental trials began the following week and were conducted during a 3-wk period. Each rat received three trials of each type. Trials were administered in blocks of three, each block including one Object trial, one Place trial, and one Context trial, in a randomly determined order. Rats received one block of trials per week, with the three trials of each block conducted on consecutive days.
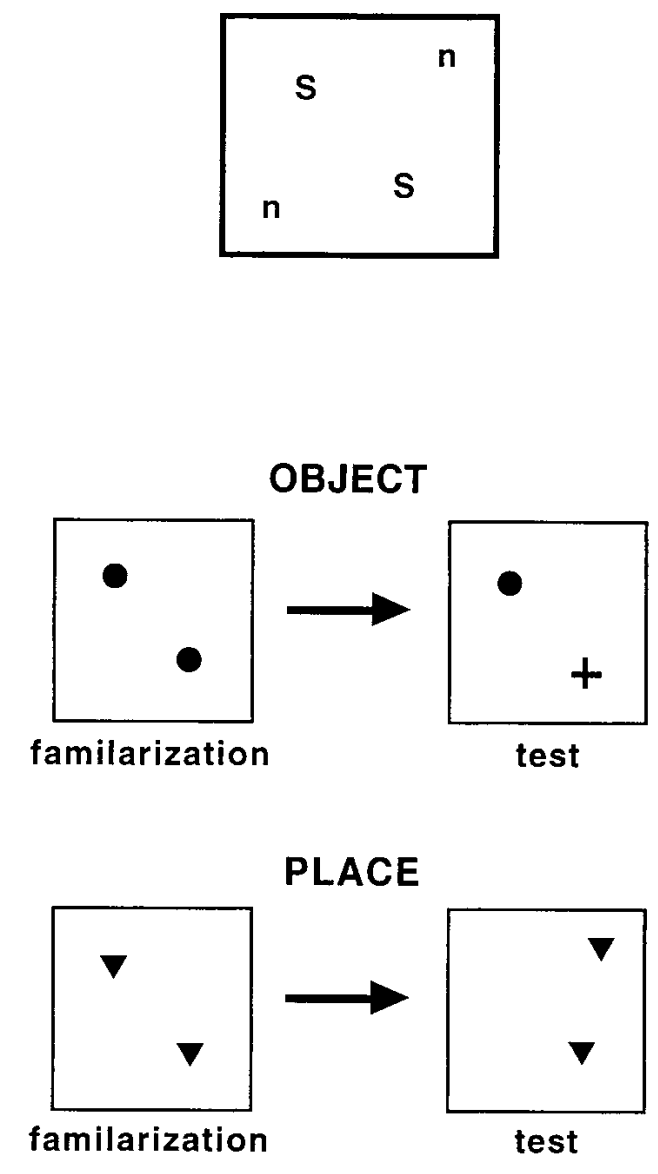

\section{CONTEXT}

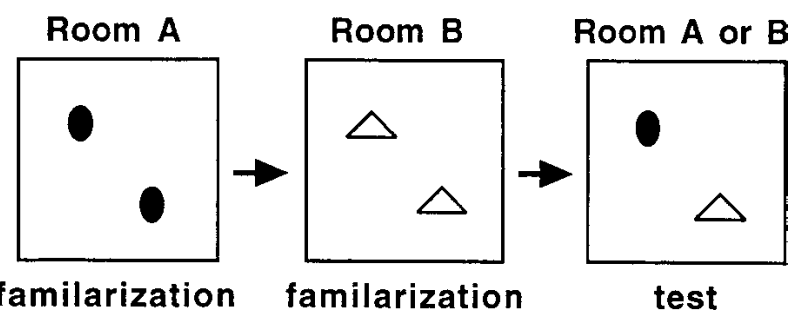

Figure 4 Top shows the relative dimensions of the open-field arenas and the positions of the stimulus objects. Objects were positioned at the standard locations (S) on Object trials and Context trials and for the familiarization phase of Place trials. One object was moved to a novel location (n) for the retention test on Place trials. Schematics illustrate the general procedure for Object, Place, and Context trials.

The particular objects for a given trial were randomly determined, but each object was used for only one trial per rat. Each HPC rat was paired with a sham rat and each pair received an identical sequence of trials, with the same objects used on corresponding trials. Thus, the groups were approximately equated for trial sequence and object pairings. The one additional sham rat was randomly assigned one of the trial sequences.

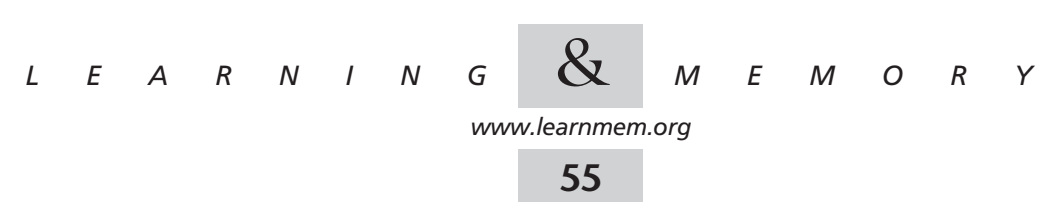


For half of the rats in each group, the first Object trial and the first Place trial were in Room A and for the other half in Room B; the testing room was reversed for the second Object and Place trials and randomly determined for the third trials. There were four novel locations that objects could be moved to on Place trials, two in each arena (Fig. 1). A different novel location was used on each of the three Place trials for a particular rat. For half of the rats in each group the retention test on the first Context trial was in Context A (Room A) and for the other half it was in Context B (Room B); the test Context was reversed for the second trial and randomly determined for the third trial. Thus, overall, each rat received roughly equal exposure to the two rooms and arenas. The counterbalancing in the Context trials also meant that on half of the trials the target object was the object from the first familiarization phase and on the other half it was the object from the second familiarization phase.

A rat was considered to be engaged in object exploration when its head was oriented within $45^{\circ}$ of an object and within $4 \mathrm{~cm}$ of it. Rearing with the head oriented upward was also included if at least one forepaw was on the object. Climbing over or sitting on the objects was not included. Trials were excluded if a rat spent less than 1 sec exploring both the sample and target object during the retention test. This necessitated the exclusion of only one trial for one rat.

The main dependent measure was the exploration ratio, that is, the proportion of total object-exploration that was spent exploring the novel object $\left(\mathrm{t}_{\text {novel }} /\left[\mathrm{t}_{\text {novel }}+\mathrm{t}_{\text {sample }}\right]\right)$ during each minute of the 3-min test phase. This minute-by-minute assessment makes it possible to see how exploratory preferences change over the retention test. Previous studies found that preference for the novel object on the object-recognition version is robust during only the first 1 or $2 \mathrm{~min}$ of a retention test and diminishes thereafter, presumably because both objects become equally familiar as they are explored (Dix and Aggleton 1999; Mumby 2001). The data were analyzed with repeated measures analyses of variance (ANOVAs) and planned comparisons to compare the performance of sham and HPC rats. Given that the main hypotheses being tested in this experiment were that HPC rats would be impaired on Place and Context trials but not on Object trials, planned $t$ tests provide the most appropriate method of making between-group comparisons on each version (Keppel 1991). To determine whether the rats discriminated between the target and sample objects, the exploration ratios obtained under each condition were compared with what would be expected by chance (i.e., a ratio of $\sim .50$ ), using one-sample $t$ tests

\section{ACKNOWLEDGMENTS}

This research was funded by the Natural Science and Engineering Research Council of Canada, and FCAR, Quebec (Grant no. 15693798).

The publication costs of this article were defrayed in part by payment of page charges. This article must therefore be hereby marked "advertisement" in accordance with 18 USC section 1734 solely to indicate this fact.

\section{REFERENCES}

Aggleton, J.P. and Brown, M.W. 1999. Episodic memory, amnesia, and the hippocampal-anterior thalamic axis. Behav. Brain Sci. 22: 425-444.

Aggleton, J.P., Hunt, P.R., and Rawlins, J.N.P. 1986. The effects of hippocampal lesions upon spatial and non-spatial tests of working memory. Behav. Brain Res. 19: 133-146.
Cassaday, H.J. and Rawlins, J.N.P. 1997. The hippocampus, objects, and their contexts. Behav. Neurosci. 111: 1228-1244.

Clark, R.E., West, A.N., Zola S.M., and Squire, L.R. 2001. Rats with lesions of the hippocampus are impaired on the delayed nonmatching-to-sample task. Hippocampus 11: 176-186.

Clark, R.E., Zola, S.M., and Squire, L.R. 2000. Impaired recognition memory in rats after damage to the hippocampus. J. Neurosci. 20: $8853-8860$.

Dellu, F., Mayo, W., Cherkaoui, J., Le Moal, M, and Simon, H. 1992. A two-trial memory task with automated recording: Study in young and aged rats. Brain Res. 588: 132-139.

Dix, S.L. and Aggleton, J.P. 1999. Extending the spontaneous preference test of recognition: Evidence of object-location and object-context recognition. Behav. Brain Res. 99: 191-200.

Duva, C.A., Floresco, S.B., Wunderlich, G.R., Lao, T.L., Pinel, J.P.J., and Phillips, A.G. 1997. Disruption of spatial but not object-recognition memory by neurotoxic lesions of the dorsal hippocampus in rats. Behav. Neurosci. 111: 1184-1196.

Duva, C.A. and Pinel, J.P.J. 1999. Functional anatomy of the medial-temporal-lobe memory system: The myth of the hippocampus. In Animal models of buman emotion and cognition (eds. M. Haug and R.E. Whalen), pp. 197-214. American Psychological Association, Washington, DC.

Eichenbaum, H. 2000. A cortical-hippocampal system for declarative memory. Nat. Rev. Neurosci. 1: 41-50.

Eichenbaum, H., Otto, T., and Cohen, N.J. 1994. Two functional components of the hippocampal memory system. Behav. Brain Sci. 17: 449-518.

Ennaceur, A. and Aggleton, J.P. 1994. Spontaneous recognition of object configurations in rats: Effects of fornix lesions. Exp. Brain Res. 100: 85-92.

Ennaceur, A. and Delacour, J. 1988. A new one-trial test for neurobiological studies of memory in rats. I: Behavioral data. Behav. Brain Res. 31: 47-59.

Ennaceur, A., Neave, N., and Aggleton, J.P. 1996. Neurotoxic lesions of the perirhinal cortex do not mimic the behavioural effects of fornix transection in the rat. Bebav. Brain Res. 80: 9-25.

-1997. Spontaneous object recognition and object location memory in rats: The effects of lesions in the cingulate cortices, the medial prefrontal cortex, the cingulum bundle and the fornix. Exp. Brain Res. 113: 509-519.

Glenn, M.J. and Mumby, D.G. 1996. Place- and object-recognition deficits following lesions of the hippocampus or perirhinal cortex in rats: A double dissociation. Society for Neuroscience Abstracts 22: 1120.

Hirsch, R. 1974. The hippocampus and contextual retrieval of information from memory: A theory. Behav. Biol. 12: 421-444.

Jackson-Smith, P., Kesner, R.P., and Chiba, A.A. 1993. Continuous recognition of spatial and nonspatial stimuli in hippocampal-lesioned rats. Behav. Neural Biol. 59: 107-119.

Jarrard, L.E. 2001. Retrograde amnesia and consolidation: Anatomical and lesion considerations. Hippocampus 11: 43-49.

Kesner, R.P., Bolland, B.L., and Dakis, M. 1993. Memory for spatial locations, motor responses, and objects: Triple dissociation among the hippocampus, caudate nucleus, and extrastriate visual cortex. Exp. Brain Res. 93: 462-470.

Keppel, G. 1991. Design and analysis: A researcher's handbook, 3rd ed. Prentice Hall, Englewood Cliffs, NJ.

Kim, J.J. and Fanselow, M.S. 1992. Modality-specific retrograde amnesia of fear. Science 256: 675-677.

Kim, J.J., Rison., R.A., and Fanselow, M.S. 1993. Effects of amygdala, hippocampus, and periaqueductal gray lesions on short- and long-term contextual fear. Behav. Neurosci. 107: 1093-1098.

Maren, S. and Fanselow, M.S. 1997. Electrolytic lesions of the fimbria/fornix, dorsal hippocampus, or entorhinal cortex produce anterograde deficits in contextual fear conditioning in rats. Neurobiol. Learn Mem. 67: 142-149.

Morris, R.G.M., Garrud, P., Rawlins, J.N.P., and O'Keefe, J. 1982. Place

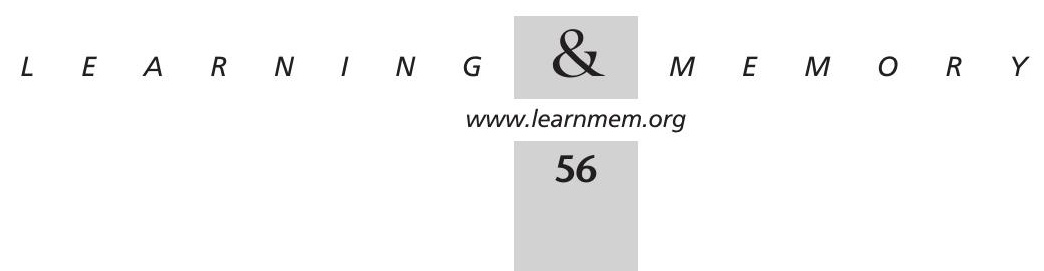


navigation impaired in rats with hippocampal lesions. Nature 297: 681-683.

Mumby, D.G. 2001. Perspectives on object-recognition memory following hippocampal damage: Lessons from studies in rats. Behav. Brain Res. 127: 159-181.

Mumby, D.G., Pinel, J.P.J., Kornecook, T.J., Shen, M.J., and Redila,V.A. 1995. Memory deficits following lesions of hippocampus or amygdala in rats: Assessment by an object-memory test battery. Psychobiology 23: $26-36$.

Mumby, D.G., Wood, E.R., Duva, C.A., Kornecook, T.J., Phillips, A.G., and Pinel, J.P.J. 1996. Ischemia-induced object-recognition deficits in rats are attenuated by hippocampal ablation before or soon after ischemia. Behav. Neurosci. 110: 266-281.

Mumby, D.G., Wood, E.R., and Pinel, J.P.J. 1992. Recognition memory in rats is only mildly impaired by lesions of the hippocampus and amygdala. Psychobiology 20: 18-27.

Nadel, L. and Moscovitch, M. 1997. Memory consolidation, retrograde amnesia, and the hippocampal complex. Curr. Opin. Neurobiol. 7: 217-227.

O'Keefe, J. and Nadel, L. 1978. The hippocampus as a cognitive map. Oxford University Press, Oxford, U.K..

Rawlins, J.N.P., Lyford, G.L., Seferiades, A., Deacon, R.M.J., and Cassaday, H.J. 1993. Critical determinants of nonspatial working memory deficits in rats with conventional lesions of hippocampus or fornix. Behav. Neurosci. 107: 420-433.

Redish, A.D. and Touretzky, D.S. 1997. Cognitive maps beyond the hippocampus. Hippocampus 7: 15-35

Rothblat, L.A. and Kromer, L.F. 1991. Recognition memory in the rat: The role of the hippocampus. Behav. Brain Res. 42: 25-32.

Save, E., Buhot, M.C., Foreman, N., and Thinus-Blanc, C. 1992. Exploratory activity and response to a spatial change in rats with hippocampal or posterior parietal cortical lesions. Behav. Brain Res. 47: 113-127.

Shaw, C. and Aggleton, J.P. 1993. The effects of fornix and medial prefrontal lesions on delayed non-matching-to-sample by rats. Behav. Brain Res. 54: 91-102.

Steckler, T., Drinkenburg, W.H.I.M., Sahgal, A., and Aggleton, J.P. 1998. Recognition memory in rats-II. Neuroanatomical substrates. Prog. Neurobiol. 54: 313-332.

Sutherland, R.J., Kolb, B.E., and Whishaw, I.Q. 1982. Spatial mapping: Definitive disruption by hippocampal or medial frontal cortical damage. Neurosci. Lett. 31: 271-276.

Warburton, E.C. and Aggleton, J.P. 1999. Differential deficits in the Morris water maze following cytotoxic lesions of the anterior thalamus and fornix transection. Behav. Brain Res. 98: 27-38.

Whishaw, I.Q., McKenna, J.E., and Maaswinkel, H. 1997. Hippocampal lesions and path integration. Curr. Opin. Neurobiol. 7: 228-234.

Wiig, K.A. and Bilkey, D.K. 1995. Lesions of rat perirhinal cortex exacerbate the memory deficit observed following damage to the fimbria-fornix. Behav. Neurosci. 109: 620-630.

Yee, B.K. and Rawlins, J.N.P. 1994. The effects of hippocampal formation ablation or fimbria-fornix section on performance of a non-spatial radial arm maze task by rats. J. Neurosci. 14: 3766-3774.

Young, S.L., Bohenek, D., and Fanselow, M.S. 1994. NMDA processes mediate anterograde amnesia of contextual fear conditioning induced by hippocampal damage: Immunization against amnesia by context preexposure. Behav. Neurosci. 108: 19-29.

Received May 30, 2001; accepted in revised form March 19, 2002.

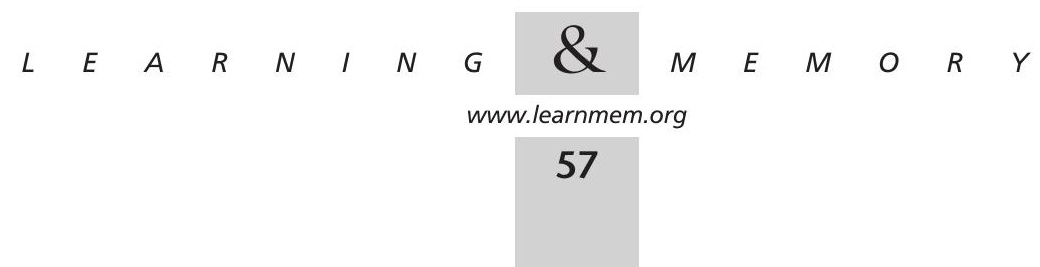




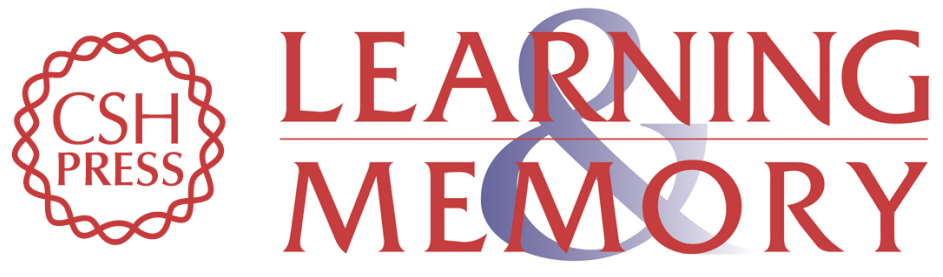

\section{Hippocampal Damage and Exploratory Preferences in Rats: Memory for Objects, Places, and Contexts}

Dave G. Mumby, Stephane Gaskin, Melissa J. Glenn, et al.

Learn. Mem. 2002, 9:

Access the most recent version at doi:10.1101/lm.41302

References This article cites 40 articles, 3 of which can be accessed free at: http://learnmem.cshlp.org/content/9/2/49.full.html\#ref-list-1

License

Email Alerting Receive free email alerts when new articles cite this article - sign up in the box at the Service top right corner of the article or click here. 